\title{
Beat Induction from Motion Capture Data Using Short-Term Principal Component Analysis
}

\author{
（短時間主成分分析を用いたモーションキャプチャデータからのビート抽出手法）
}

Jianfeng Xu (member) ${ }^{\dagger}$, Koichi Takagi (member) ${ }^{\dagger}$ and Akio Yoneyama (member) ${ }^{\dagger}$

\begin{abstract}
We propose a technique called short-term principal component analysis (ST-PCA) to analyze motion capture (MoCap) data of realistic movements in a high dimensional time series. Our ST-PCA method is successfully applied to motion beat induction, which is an important aspect in human perception. ST-PCA performs PCA in a sliding window to locally extract the major variance of the movement into the coordinates of the first principal component, thus accurately determining the desired motion beats. Our approach differs from conventional methods in that we estimate the motion beats by analyzing the motion signals as a whole rather than individually in each channel. Moreover, our algorithm is carefully designed in terms of the three characteristics of MoCap data: hierarchical structure, spatial correlation, and temporal coherence. Experimental results demonstrate that the proposed method outputs much more accurate motion beats in a wide range of motion categories, including complicated dances, than current state-of-the-art alternatives.
\end{abstract}

Key words: motion capture, beat induction, Short-Term Principal Component Analysis (ST-PCA), dimension reduction

\section{Introduction}

Motion capture (MoCap) data, which are captured from real actors, are widely applied in computer animation, video games, and movies ${ }^{1)}$, promoting significant research in understanding, organizing, and re-using motion data ${ }^{2}$. One important perception of human motion is its beat induction ${ }^{3)}$, which is essential in understanding the motion structure (especially in dances) and thus has many potential applications such as synchronization with music ${ }^{3)-6)}$. Basically, for the purpose of such an application, it is required to automatically extract motion beats from a large scale MoCap database without any manual assistance.

MoCap data, consisting of a sequence of human poses (called frames), record realistic movements in a high dimensional time series that has strong spatial and temporal correlations. Each frame is commonly modeled using a kinematic chain with high Degrees Of Freedom (DOFs) that defines a stick figure of human pose in a tree structure, see Fig. 1. Namely, each frame is in a hierarchical nature with a root joint as its origin, which controls the global translation and orientation. Assuming we have $T$ frames, MoCap data are represented as

Received September 1, 2009; Revised February 12, 2010; Accepted March 9, 2010

$\dagger$ KDDI R\&D Laboratories Inc.

(2-1-15 Ohara, Fujimino-shi, 356-8502 Saitama)

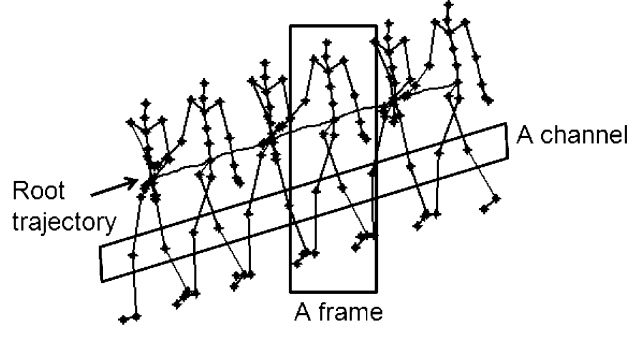

Fig. 1 A walking motion 02_01 where six sample frames and the trajectory of root joint are shown. Asterisks in stick figures denote the joint positions.

a matrix $\mathbf{H}_{D \times T}$, where $D$ denotes the DOFs in a frame and $D$ is kept as a constant in all the frames. Thus, each DOF is called a channel. Fig. 1 shows a walking motion 02_01 in CMU MoCap database ${ }^{7)}$ as an example.

Following Kim et al. ${ }^{3)}$, motion beats are defined as the regular moments when the movement is changed significantly in direction or magnitude, where the concept is obviously inspired by music ${ }^{8)}$. To estimate the motion beats, the prevailing methods are to analyze MoCap data in each channel according to some observed rules (e.g. beats happen at around zero-crossing of angular acceleration ${ }^{3)}$, sharp changes in the velocity of any body part ${ }^{4)}$, or around pause movements ${ }^{5)}$ ). According to the definition, frequency analysis such as Fourier transform is employed to further refine the motion beats in Kim et al. ${ }^{3)}$. However, the rule-based methods are 
devoid of generalization because those rules only hold in limited motion categories and are sensitive to noise. Moreover, their algorithms have difficulty in optimizing the parameters, which are set empirically and affect the results directly.

Different from the previous approaches ${ }^{3) \sim 5)}$ that analyze motion signals in each channel, we estimate the motion beats by our novel ST-PCA method that utilizes motion signals as a whole, where we avoid the difficult issue of setting the joint weights ${ }^{9)}$ that is required by Shiratori et al. ${ }^{5}$. PCA is a powerful tool to analyze multivariate data set (usually with much redundancy) ${ }^{10)}$ such as face images ${ }^{11)}$ and thus is popular in MoCap data as a pre-processing or analysis tool ${ }^{12) 13)}$. By PCA, the raw data are transformed into an uncorrelated space with a descending variability, making it possible to discard most of the dimensions without much of information loss. Therefore, PCA is widely used in dimension reduction, offering a convenient way to control the trade-off between losing information and simplifying the problem. However, PCA does not consider temporal coherence among the data samples in MoCap data. The proposed ST-PCA can, on the other hand, utilize the temporal coherence in MoCap data to improve the flexibility of its usage, which is different from the conventional usage of PCA in the literature ${ }^{10)}$.

It is observed that the major movement variance reveals motion beats well. On the other hand, PCA is well-known at extracting major variance in a multivariate data set $^{10)}$. However, in a complicated motion, the local variance is submerged in the entire variance, which infers that the conventional global PCA is inappropriate for our task. In other words, MoCap data are widely known as a highly non-linear signal, where the global PCA cannot work well ${ }^{14) \sim 16)}$. A direct solution for the above problem is called kernel PCA, which is an extension of PCA using techniques of kernel methods in a reproducing kernel Hilbert space with a non-linear mapping ${ }^{17)}$. However, as far as we know, a successful kernel function has not been reported for MoCap data yet. Recently, non-linear dimension reduction is employed in MoCap data such as Multi-Dimensional Scaling $(\mathrm{MDS})^{18)}$ and $\mathrm{ISOMAP}^{19)}$. Shin et al. ${ }^{16)}$ report a comparison among PCA, MDS, and ISOMAP in MoCap data. However, these non-linear methods such as MDS and ISOMAP are much more complex than PCA and a manual motion segmentation is even required in MSD and ISOMAP ${ }^{16)}$, which thus cannot be directly used in this paper. Another approach is to cluster data
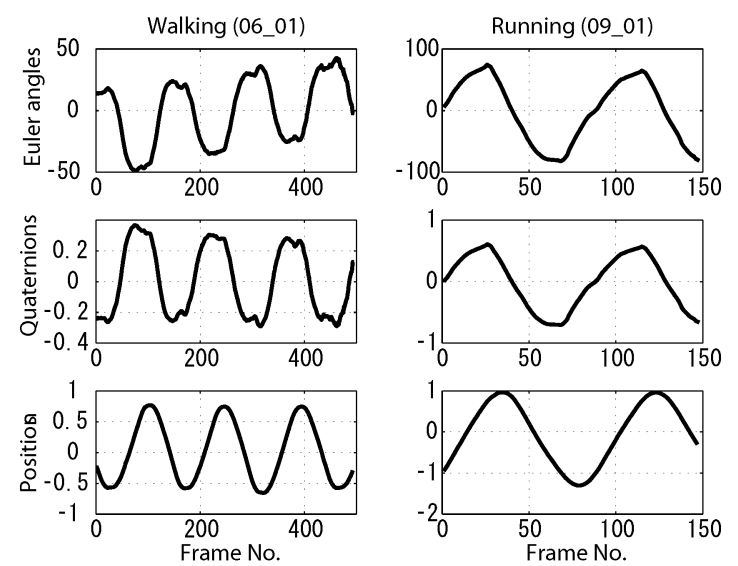

Fig. 2 The coordinates in the first principal component, calculated from Euler angles (top row), quaternions (middle row), and related joint positions (bottom row), respectively.

firstly and then perform PCA in each cluster. For example, local $\mathrm{PCA}^{20) 21)}$ uses $\mathrm{k}$-means clustering and Liu et al. ${ }^{22)}$ segment a complicated motion into single motions. However, the clustering procedure increases the computational cost greatly. Contrastively, we propose a completely automatic method without increasing much computational cost in this paper.

Our basic idea is similar to piece-wise linear approximation to a non-linear problem. PCA is a linear model while a complicated motion is highly non-linear. However, in the short term, motion data are almost linear due to the strong temporal coherence ${ }^{23)}$. Therefore, we divide the entire sequence into small motion clips and perform PCA in each clip. We will demonstrate that our proposed ST-PCA can, in a sliding window, extract very accurate motion beats not only from simple motions but also from complicated motions such as dances.

The remainder of this paper is organized as follows. In Sect. 2, we describe a pre-processing step, where the raw data in MoCap data are transformed to joint positions, which are more suitable for PCA. In Sect. 3, we present the detail procedure to extract motion beats, followed by a refinement step in Sect. 4. In Sect. 5, experimental results are reported and analyzed. Lastly, we present our conclusions and describe the future work in Sect. 6.

\section{Transformation from angles to posi- tions}

A popular way to store MoCap data is Euler angles in a local coordinate system ${ }^{7)}$, represented as $\mathbf{H}_{D \times T}$ as mentioned in Sect. 1. Such a representation is suitable to render, control, and edit MoCap data. Quaternions, 
as a non singular representation, are equivalent to Euler angles ${ }^{24)}$. However, both are not appropriate for PCA. The space of Euler angles or quaternions is non-linear in the sense of the least square terms, which is employed in PCA. Also, Euler angles or quaternions have difficulty in reflecting spatial correlation in MoCap data.

On the other hand, it is observed that joint positions in a global Cartesian coordinate system avoid effectively the above issues, which can be transformed from Euler angles using the hierarchical structure by forward kinematics ${ }^{24)}$. Because the root translation is usually the major variance but contains little beat information, related joint positions to the root joint are employed. Fig. 2 shows the coordinates in the first principal component for Euler angles, quaternions, and related joint positions, respectively, which demonstrates related joint positions have much more regular curves to reveal the cyclical motions than the other two. Note that this procedure can be regarded as a kernel function $^{17)}$, through which spatial correlation can be utilized more thoroughly. We still use a matrix $\mathbf{P}_{M \times T}$ to denote the related joint positions in human motion, where $M=3 K$ and $K$ denotes the joint number except the root joint in the human skeleton.

\section{Beat Induction by ST-PCA}

A human motion sequence may consist of many different motions (e.g. including locomotions, sports, and complicated dances). Essentially, the conventional global PCA requires a Gaussian distribution in the data, which is obviously unsuitable for such complicated data as a long human motion sequence ${ }^{16) 21) 22)}$. On the other hand, the data in the short term yield approximately a Gaussian distribution. Therefore, it is necessary to perform PCA in the short term. Liu et al. ${ }^{22)}$ segment a complicated motion into single motions by Barbič's algorithm ${ }^{12)}$. In this paper, we have observed that PCA works well in a sliding window with a fixed length, thus removing the heavy processing of segmenting as Liu et al.' $\mathrm{s}^{22)}$. In the short term, motion has strong temporal coherence, making it possible to obtain the movement variance by the data in a window. Fig. 3 demonstrates that we can obtain almost the same coordinates as the global PCA in a single motion no matter how long the window is. Therefore, it is not necessary to know the boundary of a single motion in the case of a multimotion sequence if the window length is shorter than the single motion, allowing a fixed-length window effective. This characteristic forms the base of our ST-PCA

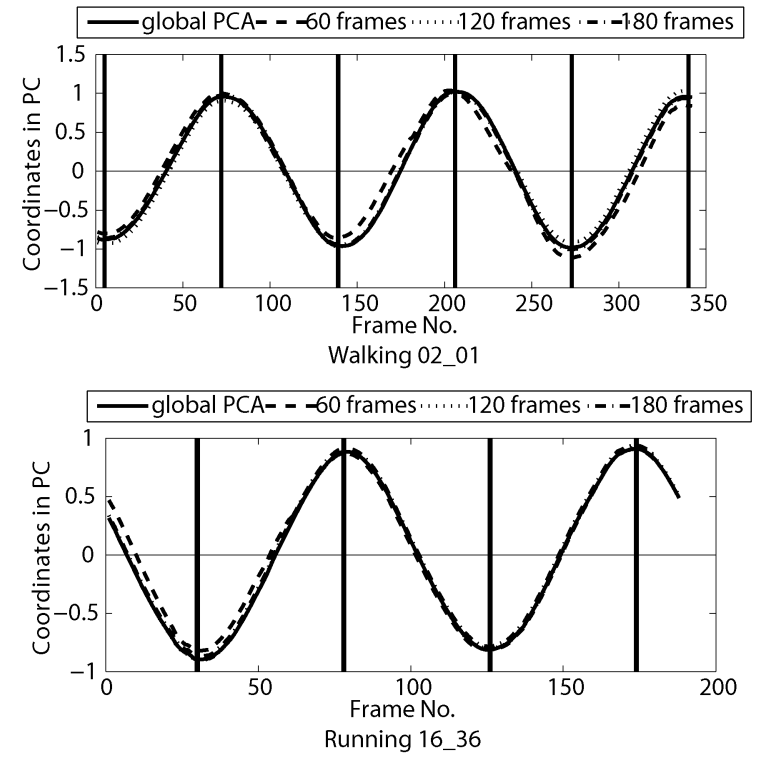

Fig. 3 The coordinates of the first principal component in Walking 02_01 (top) and Running 16_36 (bottom), calculated by different window lengths (see the legend), and vertical lines denote the ground truth of beats..

algorithm, where PCA is performed in a fixed-length sliding window.

By dividing the entire motion $\mathbf{P}_{M \times T}$ using a sliding window with $N$ frames, we obtain a set of matrices $\left\{\mathbf{X}_{M \times N}^{i}: i=1, \ldots, I\right\}$, where $\mathbf{X}_{M \times N}^{i}$ is a submatrix of $\mathbf{P}_{M \times T}$ that consists of columns from $(i-1) N+1$ to $i N^{*}$ and $I=\lceil T / N\rceil$, denoting the number of windows.

In each window, we reduce the motion data $\mathbf{X}_{M \times N}^{i}$ (briefly $\mathbf{X}^{i}$ ) to one dimension by PCA. After removing the average $\overline{\mathbf{X}}^{i}$ in $\mathbf{X}^{i}$, singular value decomposition $(\mathrm{SVD})$ is used to perform PCA on $\mathbf{D}^{i}=\left(\mathbf{X}^{i}-\overline{\mathbf{X}}^{i}\right)^{T}$ as

$$
\mathbf{D}^{i}=\mathbf{U}^{i} \Lambda^{i} \mathbf{V}^{i T}
$$

where columns of $\mathbf{U}^{i}$ and $\mathbf{V}^{i}$ are orthogonal unit vectors, and $\boldsymbol{\Lambda}^{i}$ is a diagonal square matrix with nonnegative decreasing singular values on its diagonal. Columns of $\mathbf{V}^{i}=\left\{\mathbf{v}_{1}^{i}, \ldots, \mathbf{v}_{M}^{i}\right\}$ form the bases of transformation to principal component space.

As described before, we only use the coordinates in the first principal component $\mathbf{y}_{1}^{i}$ to estimate motion beats, which can be obtained by

$$
\mathbf{y}_{1}^{i}=\left(\mathbf{D}^{i} \mathbf{v}_{1}^{i}\right)^{T}
$$

From another point of view, the first basis $\mathbf{v}_{1}^{i}$ can be regarded as joint weights for the $i$-th window, which is automatically optimized by PCA in Eq.(1). By $\mathbf{v}_{1}^{i}$, we can obtain the major movement variance $\mathbf{y}_{1}^{i}$ in the $i$-th

${ }^{*}$ For $\mathbf{X}_{M \times N}^{I}$ in the last window, the frames may be less than $N$. 


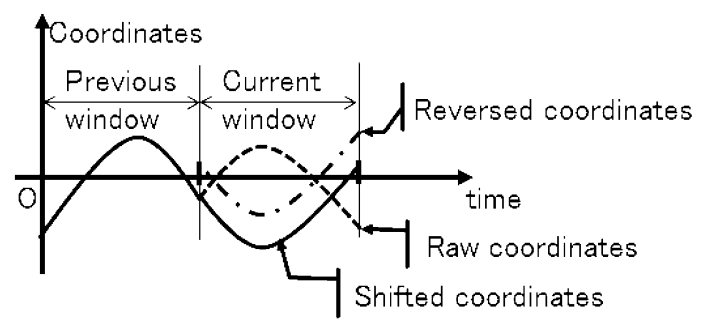

Fig. 4 The coordinates of the first principal component in the current window are connected smoothly with those in the previous window by reversal and shifting operations.

window from the original motion data $\mathbf{D}^{i}$. Compared to other methods such as Shiratori et al. ${ }^{5}$, our method does not need to set the joint weights, thus requiring no prior knowledge of motions. Moreover, it is natural that joint weights $\mathbf{v}_{1}^{i}$ will vary in different windows to match the motion contents, leading to ST-PCA rather reasonable.

Discontinuity happens at the boundary between two windows due to the independent operation of ST-PCA in each window. Therefore, it is necessary to connect the coordinates in the current window to those in the previous window, where two steps are performed as shown in Fig. 4. Firstly, we will determine if the basis of the first principal component in the current window is consistent with that in the previous window. Basically, the two bases in the neighboring windows should not change very sharply due to the temporal coherence. Therefore, we reverse the basis in the current window by $\mathbf{v}_{1}^{i^{\prime}}=-\mathbf{v}_{1}^{i}$, guaranteeing the angle between two bases is always smaller than $\pi / 2^{*}$, if inner product of two bases is negative as

$$
\mathbf{v}_{1}^{i} \cdot \mathbf{v}_{1}^{(i-1)^{\prime}}<0
$$

where $\mathbf{v}_{1}^{i}$ denotes the first basis in the current window before the reversal operation, and $\mathbf{v}_{1}^{(i-1)^{\prime}}$ denotes the first basis in the previous window after the reversal operation. For the first window, it is no need to perform the reversal operation.

Corresponding to the basis, the coordinates in the current window have the similar operation $\mathbf{y}_{1}^{i^{\prime}}=-\mathbf{y}_{1}^{i}$ if Eq. (3) is satisfied.

Then, according to the coordinates of the last frame in the previous window and the first frame in the current window, we shift properly all the coordinates in the current window to obtain the connected coordinates

\footnotetext{
* Note that in MoCap data, the phase difference should not be and has not been omitted by the reversal operation since PCA itself does not change the phase of the motion signal.
}

$\mathbf{y}_{1}^{i^{\prime \prime}}=\left\{y_{1}^{i^{\prime \prime}}(1), \ldots, y_{1}^{i^{\prime \prime}}(N)\right\}$ in the current window as

$$
\mathbf{y}_{1}^{i^{\prime \prime}}=\mathbf{y}_{1}^{i^{\prime}}+\left(y_{1}^{(i-1)^{\prime \prime}}(N)-y_{1}^{i^{\prime}}(1)\right) * \mathbf{I}_{N},
$$

where $\mathbf{y}_{1}^{i}$ denotes all the coordinates of the first principal component in the $i$-th window, $y_{1}^{i}(k)$ denotes the $k$-th coordinate of the first principal component in the $i$-th window, $\mathbf{I}_{N}$ denotes the $\mathrm{N}$ dimensional vector with the element of $1,{ }^{\prime}$ denotes the values after the reversal operation, and " denotes the values after the reversal and shifting operations. Note that the shifting operation changes no phase difference between two windows, resulting that phase difference is kept after reversal and shifting operations.

It is ready to estimate motion beats after obtaining the coordinates that are connected smoothly among neighboring windows. Those frames with local extreme values $C B=\{c b(j): j=1, \ldots, J\}$ are regarded as a set of coarse beat moments, which will be refined in next section.

ST-PCA has the same computational cost as the global PCA except the connection operation (whose complexity is much less than PCA's), because computational complexity of PCA is $O\left(M^{2} \times N\right)$, which is linear to the frame number $N$. Furthermore, ST-PCA is suitable for parallel computing with the potential of high speed-up and the same memory consumption as global PCA. In the case of non-parallel implementation, it requires much less memory than global PCA.

On the other hand, ST-PCA extracts successfully the local major movement variance that reveals beat information while the global PCA fails. Fig. 5 shows the results in a running motion on a circle path, which demonstrates ST-PCA achieves a much more regular curve that reveals accurately the motion cycles existing in the motion. In the case of more complicated motions, the advantage of ST-PCA becomes even larger. It is also observed the movement variance in ST-PCA is larger than that in the global PCA (see Fig. 5 as an example).

\section{Beat Refinement}

Based on the coarse beat set $C B$, we regularize the beat intervals globally, assuming they are invariable in a motion, which is reasonable if the motion is not so long (e.g. less than 30 seconds) ${ }^{3)}$. We adopt a similar technique to Kim et al.' $\mathrm{s}^{3)}$, where frequency is analyzed in the estimated coarse beat set $C B$. Hopefully, it will alleviate the influence of errors that come from the limited samples in each window. 

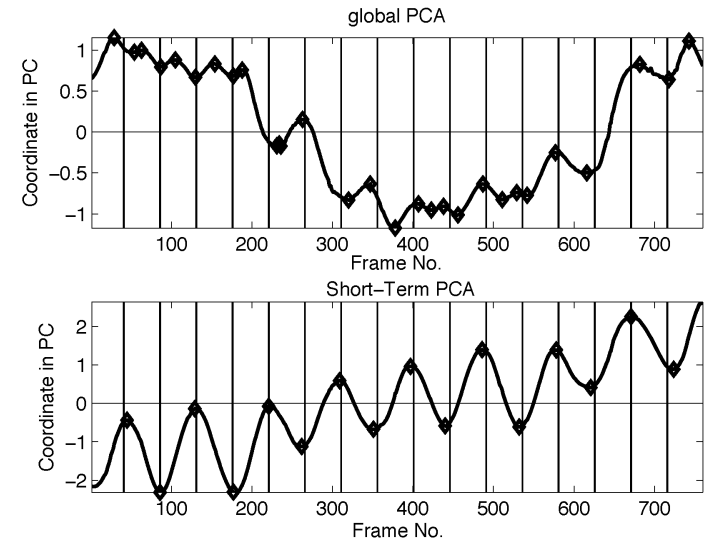

Fig. 5 The coordinates of the first principal component in a running motion on a circle path (38_03), calculated by global PCA (top) and ST-PCA (bottom). Diamonds denote the extreme values, and vertical lines denote the ground truth of beats. ST-PCA extracts local movement variance successfully while global PCA fails.

Firstly, a sinusoidal function $s(t)$ is constructed among all the beat intervals in $C B$ as

$s(t)=\cos \left(2 \pi \frac{t-c b(j-1)}{c b(j)-c b(j-1)}\right) c b(j-1) \leqq t \leqq c b(j) .(5)$

Secondly, Fourier transform is performed with a Hanning window on $s(t)$ to get the maximal frequency $f_{\max }$. Thus, the beat interval is estimated as

$$
T B=F s / f_{\max },
$$

where $F s$ is the frame rate in MoCap data.

Then, another sinusoidal function $s^{\prime}(t)$ is constructed as

$$
s^{\prime}(t)=\cos (2 \pi t / T B) c b(1) \leqq t \leqq c b(J)
$$

to estimate the phase $\hat{\theta}$ by maximizing the correlation between $s(t)$ and $s^{\prime}(t+\theta)$ as

$$
\hat{\theta}=\arg \max _{\theta} \sum_{t} s(t) s^{\prime}(t+\theta) 0 \leqq \theta<T B .
$$

Finally, we can achieve the estimated motion beats $F B$ by

$$
f b(j)=\hat{\theta}+j \cdot T B
$$

where $f b(j)$ denotes the $j$-th motion beat. Similar to music, we can count the tempo of a motion as the beat number in a minute by

$$
\text { tempo }=60 F s / T B \text {. }
$$

\section{Experimental Results}

MoCap data in our experiments are obtained from CMU Motion Capture Database ${ }^{7}$, where there are 31
Table 1 Precision and recall in a Salsa dancing motion (61_01).

\begin{tabular}{c|c|c|c|c}
\hline \hline & \multicolumn{2}{|c|}{ before refinement } & \multicolumn{2}{c}{ after refinement } \\
\hline & precision & recall & precision & recall \\
\hline Weight Effort & 0.36 & 0.36 & 0.35 & 0.27 \\
\hline global PCA & 0.48 & 0.45 & 0.47 & 0.25 \\
\hline ST-PCA & 0.84 & 0.76 & 0.91 & 1.00 \\
\hline
\end{tabular}

joints with 62 DOFs in a frame and motions are captured in 120 frames per second. We evaluate two kinds of motions including the periodic motions (e.g. walking and running) and dances. The window length in STPCA is set as 60 frames. We also compare the results with the global PCA and Shiratori et al.'s algorithm (denoted by Weight Effort later) ${ }^{5}$, where Weight Effort features in Laban's theory are employed to calculate the excitement of the motion. In more detail, weight effort is defined as the weighted sum of approximated instantaneous momentum magnitude calculated from the stick directions and global orientation. Since there are no reports on the joint weights in the reference ${ }^{5)}$, we adopt the optimized joint weights in Wang et al. ${ }^{9)}$. In both the conventional methods, we refine the motion beats as Sect. 4 to regularize the beat intervals. To evaluate the performance, the ground truth is extracted by human subjects for each motion.

As an example of complicated dances, Fig. 6 shows the estimated beats in part of a Salsa dance. Compared to the conventional methods, our method estimates much more accurate beats with a more regular curve, i.e., both the time instant and the beat interval are much nearer to the ground truth in our method. Table 1 shows the precision and recall before and after the refinement for the above Salsa dance, where the correct beats are defined as those detected beats whose absolute difference from the ground truth is shorter than $1 / 5$ of beat interval (0.05 seconds) *. As you can see, our method has much better performance than the conventional methods in a complicated dance no matter before or after the refinement. Furthermore, it is clear that the refinement improves the recall greatly because of the regular curve in our method.

Fig. 7 shows the estimated tempi from 13 motions including walking, running, and some dancing motions, which demonstrates our method is rather accurate and has better performance than both Weight Effort and the global PCA, i.e., the error from the ground truth is much smaller in our method. In detail, the average

\footnotetext{
*We adopt the same "error window" as the evaluation of audio beat tracking in MIREX $2006^{25)}$
} 
Weight Effort
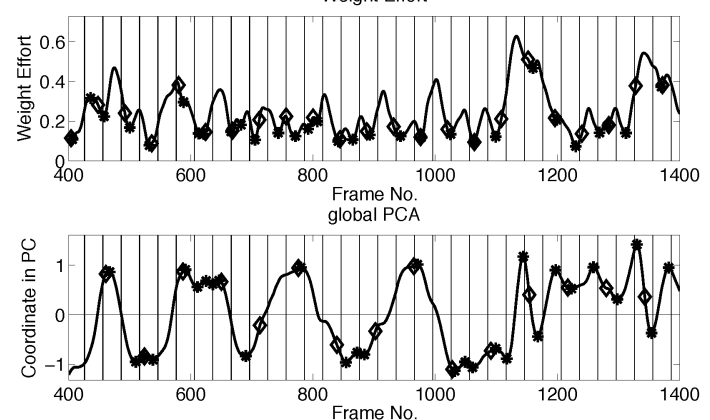

Frame No.
ST-PCA (Proposal)

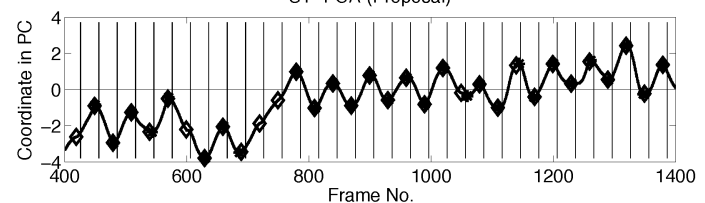

Fig. 6 Estimated beats in part of a Salsa dancing motion (61_01). Weight Effort is shown in the top rows, global PCA is shown in the middle rows, and the proposal (ST-PCA) is shown in the bottom rows. Asterisks denote the coarse beats, diamonds denote the refined beats, and vertical lines denote the ground truth of beats.

of relative errors from the ground truth is $6.67 \%$ in our method vs. $43.3 \%$ in Weight Effort and $24.2 \%$ in the global PCA.

However, our method has limitations in those motions where the major movement variance does not reveal beat information. For example, in Charleston 93_06, the body rotation becomes the major movement variance while the leg movements reveal the motion beats. Although such cases are found rarely, we observe that the coordinates in the second principal component can solve the problem.

\section{Conclusions}

In this paper, our main contribution is to propose a novel ST-PCA method to analyze MoCap data, which are a high dimensional time series with strong spatial and temporal correlations in a tree structure. Based on the fact that MoCap data have strong temporal coherence in the short term, we demonstrate that PCA in a fixed-length sliding window is effective to extract motion beats in a wide range of motion categories including complicated dances.

Different from the previous approaches ${ }^{3) \sim 5}$ that analyze motion signals in each channel, we estimate the motion beats by our ST-PCA method that utilizes motion signals as a whole. Our method extracts the local major variance in MoCap data using ST-PCA, which improves the robustness and accuracy of our algorithm and requires less parameters. Moreover, ST-PCA has the same computational complexity as the global PCA and is suitable for parallel computing. Furthermore, it can be easily extended to target just a few particular channels such as only the DOFs in legs. It is also possible to apply ST-PCA in other applications such as primitive motion extraction, key pose extraction, and motion compression ${ }^{22}$ and even in other media such as video and audio.

In the future, we plan to develop a music synchronization system based on our beat induction algorithm, which can automatically generate a new motion from a motion database that is synchronized with a piece of input music, see our preliminary result in ${ }^{6)}$.

Finally, the authors would like to thank Dr. Ryoichi Kawada, Dr. Haruhisa Kato, and Mr. Yusuke Uchida for their fruitful discussions, and the volunteers for creating the ground truth in the evaluation. The data used in this project were obtained from mocap.cs.cmu.edu ${ }^{7)}$. The database was created with funding from NSF EIA0196217.

\section{〔References〕}

1) C. Bregler: "Motion Capture Technology for Entertainment", IEEE Signal Processing Magazine, 24, 6, pp.160-158 (Nov. 2007)

2) C. Faloutsos, J. Hodgins, and N. Pollard: "Database Techniques with Motion Capture", ACM SIGGRAPH 2007 Course \#21 Notes (Aug. 2007)

3) T.H. Kim, S.I. Park, and S.Y. Shin: "Rhythmic-Motion Synthesis based on Motion-Beat Analysis", ACM Transactions on Graphics, 22, 3, pp.392-401 (Jul. 2003)

4) G. Alankus, A.A. Bayazit, and O.B. Bayazit: "Automated Motion Synthesis for Dancing Characters", Computer Animation and Virtual Worlds, Special Issue: CASA 2005, 16, 3-4, pp.259271 (2005)

5) T. Shiratori, A. Nakazawa, and K. Ikeuchi: "Dancing-to-Music Character Animation," Computer Graphics Forum, 25, 3, pp.449458 (2006)

6) J. Xu, K. Takagi, and R. Kawada: "Automatic Composition of Motion Capture Animation for Music Synchronization", Proceedings of EUROGRAPHICS 2010, short paper, to appear (2010)

7) CMU, http://mocap.cs.cmu.edu/. CMU MoCap Database (2003)

8) W. Berry, Structural Functions in Music, Dover Publications (1987)

9) J. Wang and B. Bodenheimer: "An Evaluation of a Cost Metric for Selecting Transitions Between Motion Segments", SCA '03. Proceedings of the 2003 ACM SIGGRAPH/Eurographics Symposium on Computer Animation, pp.232-238 (2003)

10) I. Jolliffe, Principal Component Analysis, Series: Springer Series in Statistics, 2nd ed. (2002)

11) M. Turk and A. Pentland: "Eigenfaces for Recognition", Journal of Cognitive Neuroscience, 3, 1, pp.71-86 (1991)

12) J. Barbič, A. Safonova, J.Y. Pan, C. Faloutsos, J.K. Hodgins, and N.S. Pollard: "Segmenting Motion Capture Data into Distinct Behaviors", GI '04: Proceedings of Graphics Interface 2004, pp.185-194 (2004)

13) R. Urtasun, P. Glardon, R. Boulic, D. Thalmann, and P. Fua: "Style-based Motion Synthesis", Computer Graphics Forum, 23, 4, pp.799-812 (2004)

14) V. Pavlovic, J.M. Rehg, and J. MacCormick: "Learning Switching Linear Models of Human Motion", Proceedings of The Neural Information Processing Systems (NIPS) Foundation, pp.981-987 (2000)

15) Y. Li, T.Wang, and H.Y. Shum: "Motion Texture: a Two-Level Statistical Model for Character Motion Synthesis", ACM Trans- 


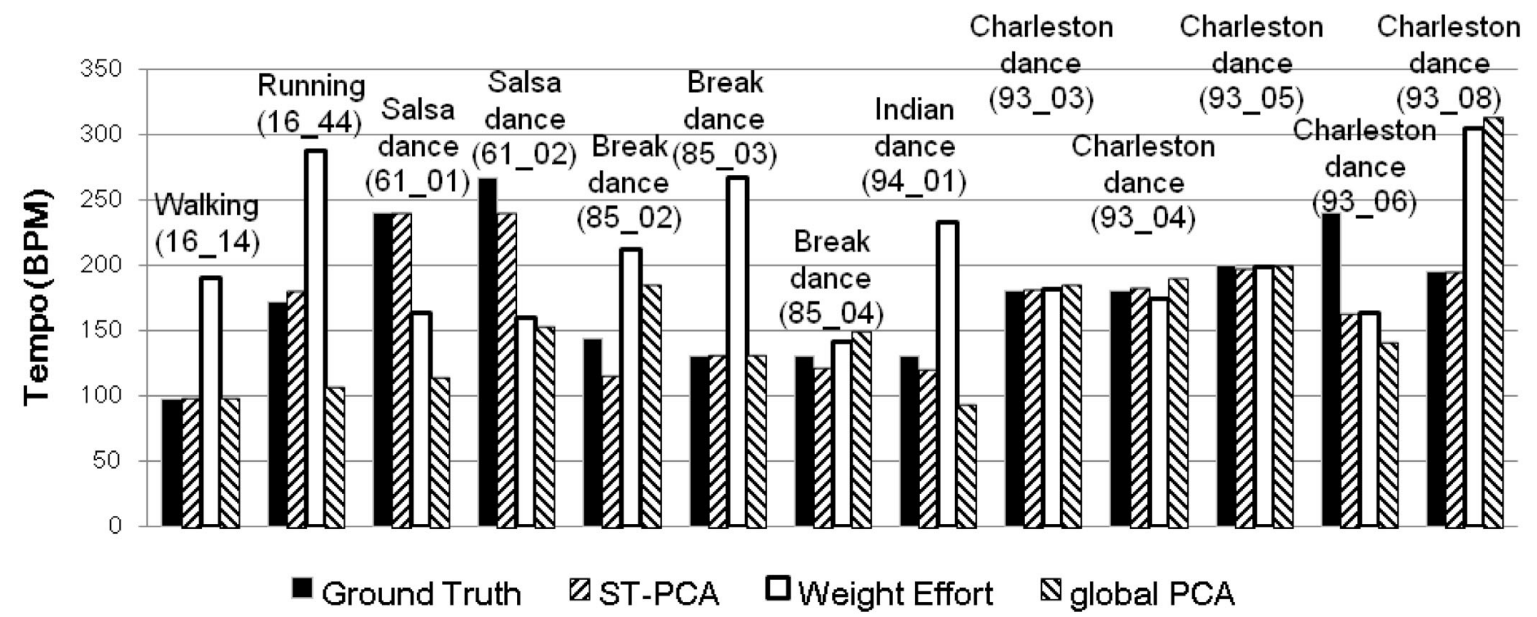

Fig. 7 Estimated tempi in walking, running, and some dancing motions including Salsa, Break, Indian, and Charleston.

actions on Graphics, 21, 3, pp.465-472 (2002)

16) H.J. Shin and J. Lee: "Motion Synthesis and Editing in Low Dimensional Spaces", Computer Animation and Virtual Worlds (Special Issue: CASA 2006), 17, 3-4, pp.219-227 (2006)

17) B. Scholkopf, A.J. Smola, and K.R. Muller: "Nonlinear Component Analysis as a Kernel Eigenvalue Problem", Neural Computation, 10, 5, pp.1299-1319 (1998)

18) I. Borg and P. Groenen, Modern Multidimensional Scaling: Theory and Applications, Springer-Verlag New York (2005)

19) J.B. Tenenbaum, V. de Silva, and J.C. Langford: "A Global Geometric Framework for Nonlinear Dimensionality Reduction", Science, 290, 5500, pp.2319-2323 (2000)

20) N. Kambhatla and T.K. Leen: "Dimension Reduction by Local Principal Component Analysis", Neural Computation, 9, 7, pp.1493-1516 (1997)

21) M.Sattler, R. Sarlette, and R. Klein: "Simple and Efficient Compression of Animation Sequences", SCA '05: Proceedings of the 2005 Eurographics/ACM SIGGRAPH Symposium on Computer Animation, pp.209-217 (2005)

22) G. Liu and L. McMillan: "Segment-Based Human Motion Compression", SCA '06: Proceedings of the 2006 ACM SIGGRAPH/Eurographics symposium on Computer Animation, pp.127-135 (2006)

23) J. Xu, T. Yamasaki, and K. Aizawa: "Summarization of 3D Video by Rate-Distortion Trade-off", IEICE Transactions on Information and Systems, E90-D, 9, pp.1430-1438 (2007)

24) D.H. Eberly, 3D Game Engine Design : A Practical Approach to Real-Time Computer Graphics (2nd Edition), Morgan Kaufmann, 2007.

25) MIREX, http://www.music-ir.org/mirex/2006/index.php/Audio_ Beat_Tracking (2009)

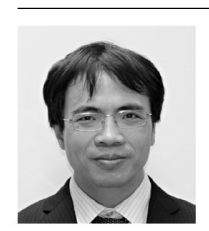

Jianfeng $\mathrm{Xu}$ received the B.S. (with honor) and the M.S. degrees from Tsinghua University, China, in 2001 and 2004 respectively and the Ph.D. degree from The University of Tokyo, Japan, in 2007. Currently, he works at KDDI R\&D Laboratories Inc. His research interests include generation, analysis, and re-use of motion capture data.

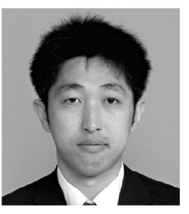

Koichi Takagi received his M.E. degree from the Tokyo Institute of Technology in 1998. He has been working at Kokusai Denshin Denwa since 1998 and now is a research engineer in the Media Solutions Laboratory at KDDI R\&D Laboratories Inc. He has been engaged in research on video coding and is currently interested in audio coding and processing. He is a member of ITE.

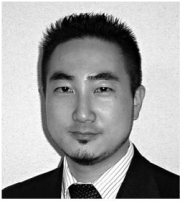

Akio Yoneyama received the B.E. and the M.E. degrees in electrical engineering from Keio University, Japan in 1992 and 1994, respectively, and the Ph.D degree in Information Processing from Tokyo Institute of Technology, Japan, in 2007. He joined KDD in 1994. Since 1996, he has been with KDDI R\&D Laboratories Inc. From 2002 to 2003, he was a visiting researcher at University of Southern California. His current research interests include visual media processing. 